\title{
FLUKA Monte Carlo for Basic Dosimetric Studies of Dual Energy Medical Linear Accelerator
}

\author{
K. Abdul Haneefa, ${ }^{1}$ T. Siji Cyriac, ${ }^{2,3}$ M. M. Musthafa, ${ }^{4}$ R. Ganapathi Raman, ${ }^{3}$ V. T. Hridya, \\ A. Siddhartha, ${ }^{6}$ and K. K. Shakir ${ }^{6}$ \\ ${ }^{1}$ Department of Physics, University \& INFN of Torino, Via P.Giuria, 10125 Torino, Italy \\ ${ }^{2}$ Department of Radiation Therapy, HCG-Dr. Balabhai Nanavati Hospital, Vile Parle (West), Mumbai 400 056, India \\ ${ }^{3}$ Department of Physics, Noorul Islam Centre for Higher Education, Kumaracoil, Thuckalay, Kanyakumari District, \\ Tamilnadu 629 180, India \\ ${ }^{4}$ Department of Physics, University of Calicut, Thenhipalam, Kerala 673635, India \\ ${ }^{5}$ Department of Radiation Therapy, K.S Hegde Medical Academy, Nitte University, Deralakatte, Mangalore, Karnataka 575018, India \\ ${ }^{6}$ Department of Radiation Therapy, AJ Cancer Institute-Kuntikana, Mangalore, Karnataka 575004, India
}

Correspondence should be addressed to K. Abdul Haneefa; abdul.haneefa@gmail.com

Received 29 January 2014; Revised 9 July 2014; Accepted 9 July 2014; Published 24 July 2014

Academic Editor: Tibor Major

Copyright (C) $2014 \mathrm{~K}$. Abdul Haneefa et al. This is an open access article distributed under the Creative Commons Attribution License, which permits unrestricted use, distribution, and reproduction in any medium, provided the original work is properly cited.

General purpose Monte Carlo code for simulation of particle transport is used to study the basic dosimetric parameters like percentage depth dose and dose profiles and compared with the experimental measurements from commercial dual energy medical linear accelerator. Varian Clinac iX medical linear accelerator with dual energy photon beams ( 6 and $15 \mathrm{MV})$ is simulated using FLUKA. FLAIR is used to visualize and edit the geometry. Experimental measurements are taken for $100 \mathrm{~cm}$ source-to-surface (SSD) in $50 \times 50 \times 50 \mathrm{~cm}^{3}$ PTW water phantom using $0.12 \mathrm{cc}$ cylindrical ionization chamber. Percentage depth dose for standard square field sizes and dose profiles for various depths are studied in detail. The analysis was carried out using ROOT (a DATA analysis frame work developed at CERN) system. Simulation result shows good agreement in percentage depth dose and beam profiles with the experimental measurements for Varian Clinac iX dual energy medical linear accelerator.

\section{Introduction}

Monte Carlo (MC) method has become a powerful tool in radiation therapy for studying the dosimetric parameters. It is a common objective of medical physics to achieve an accuracy of better than $\pm 5 \%$ for the delivery of dose. But this can be realized only if the dose calculation accuracy is better than $\pm 2 \%$. Therefore, in the future, Monte Carlo algorithms will have a clear preference compared with all other methods of dose calculation. At present, different Monte Carlo codes are used widely for modeling medical linear accelerators [1-4].

Monte Carlo methods are applied in radiation therapy to analyze the adequacy of linac head components, to benchmark dose calculation models, and to study the beam characteristics $[5,6]$. Modeling of medical linear accelerator in clinical environments using any Monte Carlo models is quite complex to address source definition and collimator materials definitions. Commercial manufactures of medical linear accelerators are distributing limited versions with confidence of agreements. Different approaches are suggested in many literatures to quantify this limited knowledge for modeling $[7,8]$. Percentage depth dose (PDD) and beam profiles are the basic parameters to ensure the adequacy of Monte Carlo modeling [9].

Monte Carlo for treatment planning is practically impossible in Indian scenarios, where there are a large number of patients with limited facilities. A Monte Carlo model takes considerable time to optimize individual treatment plans. However, the beam characteristic and other parameters can 
be tuned using any available Monte Carlo codes for all clinical institutions [10]. Time consuming process for Monte Carlo calculations is greatly influenced in our study to make simple and reliable model to mimic the clinical scenario.

In this study, we modeled a commercial medical linear accelerator, Varian Clinac iX, using FLUKA [11, 12] Monte Carlo code by using FLAIR [13] (FLUKA Advanced Interface) as per the manufactures recommendations. Percentage depth dose (PDD) values and lateral profiles of dose deposition were evaluated for standard square field sizes. Our results are compared with the experimental data taken from Varian Clinac iX machine. CERN developed ROOT v5-34 [14] software is used for analysis.

\section{Materials and Methods}

2.1. FLUKA Monte Carlo Calculations. Dual energy photon beam from Varian Clinac iX (Varian Medical Systems, Palo Alto, CA, USA) is modeled using FLUKA Monte Carlo. We simplified this model without altering any components in the linac head as per manufactures description. Version 2011.2b.4 (updated in 11 September 2013) of FLUKA code was utilized in the present research. This code is a multipurpose Monte Carlo code which has been developed for accurate simulation of the interaction and propagation in matter of about 60 different particles, including photons and electrons from $1 \mathrm{keV}$ to thousands of $\mathrm{TeV}$, neutrinos, muons of any energy, hadrons of energies up to $20 \mathrm{TeV}$ and all the corresponding antiparticles, neutrons down to thermal energies, and heavy ions. A welldescribed physical phenomenon in our energy range is the power of this tool.

Figure 1 shows major components including target, primary collimator, fattening filter, and secondary collimator jaws. Actual components combination is not shown in the figure, because of confidence of agreement signed with the manufacturer. Target with tungsten coupled with copper is defined in the simulation. Primary and secondary collimators are defined using tungsten. Water phantom of $50 \times 50 \times$ $50 \mathrm{~cm}^{3}$ cube is defined at $100 \mathrm{~cm}$ from the target with $1 \mathrm{~cm}$ plastic outer covering layer except in beam entrance plane.

FLAIR gives wide options to choose desired FLUKA input cards (the type of interaction and transport thresholds can be set in the physics and transport section of the cards). EMCASCA card is used as a default in both cases. Number of primary photons for this study was set at $5 \times 10^{7}$ for $6 \mathrm{MV}$ and $8 \times 10^{7}$ for $15 \mathrm{MV}$ histories in 5 cycles. Electromagnetic intersection input file was carried out in electromagnetic FLUKA cascade mode [12]. A production threshold of $10 \mathrm{keV}$ is used for EMF and $50 \mathrm{keV}$ for delta ray production.

2.2. Dose Measurements. All the measurements were carried out by Scanditronix automatic water phantom (Blue Phantom, Scanditronix Wellhofer AB, Sweden) and a cylindrical ionization chamber of $0.12 \mathrm{~cm}^{3}$ active volume. Both PDD curves and beam profiles were taken using $1 \mathrm{~mm}$ step size for measurements. For comparison $2 \mathrm{~mm}$ readings were considered to obtain better illustration in PDD and $1 \mathrm{~mm}$

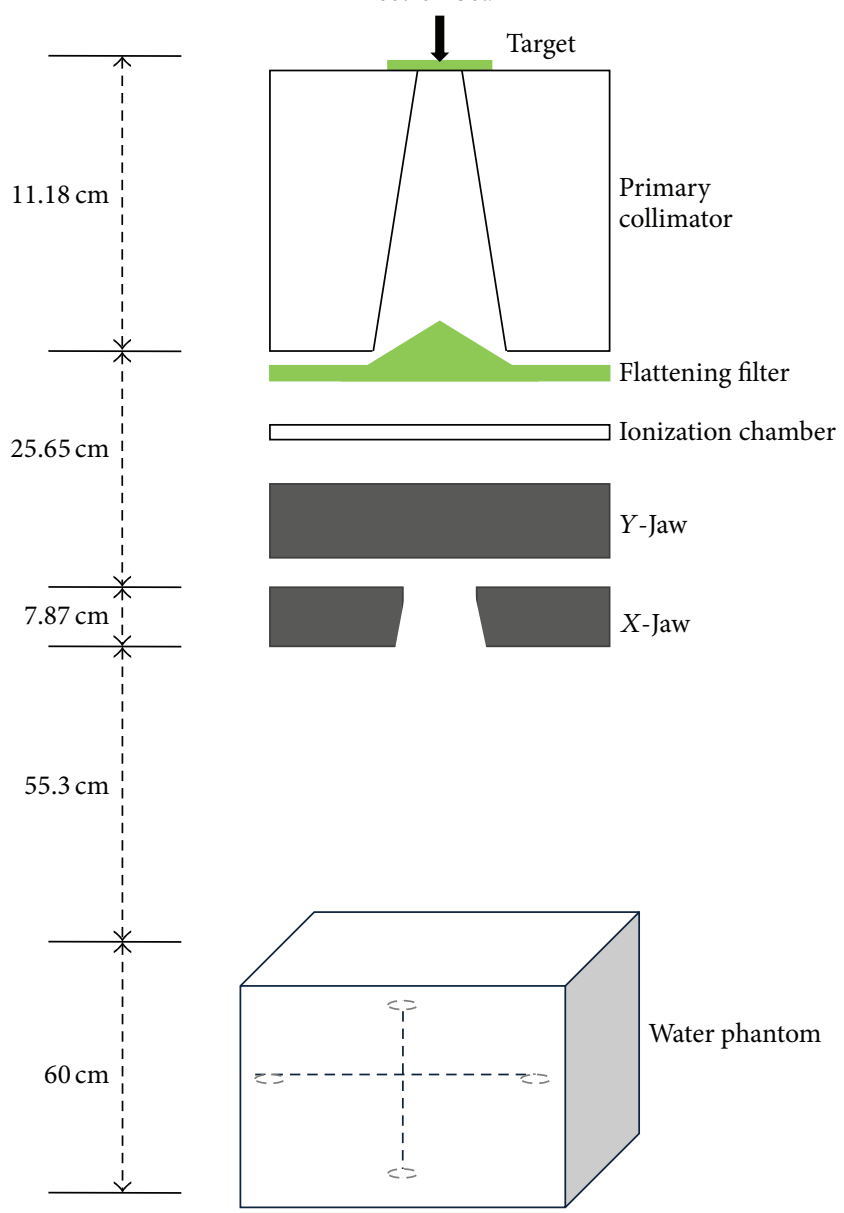

FIGURE 1: The schematic representation of Varian Clinac iX medical linear accelerator and water phantom simulated in our study.

binning for profile plots. Beam quality measurements were carried out using TPS 20/10 on both energies before the data taking. Room lasers and standard scalars are used for accurate position of water phantom.

2.3. Beam Energy Optimization. Standard $10 \times 10 \mathrm{~cm}^{2}$ field size is used to obtain the beam energy for both photon energies. Percentage depth dose for this field size is compared between Monte Carlo calculated and the measurements. This is a common approach by many authors $[1,9]$ for beam energy optimization.

Three independent measurements were taken to reduce the uncertainties in maximum dose point and the comparison is performed to this normalized point. Initial energy value of photon beam is taken as the default value as set by the manufacturer. Further, the value was increased or decreased according to the result of the first comparison between calculations and measurements. In $6 \mathrm{MV}$ photon beam, the energies of $6.0,6.2,6.3$, and $6.4 \mathrm{MeV}$ were simulated and for $15 \mathrm{MV}$ photon beam, the energies of 15.0 , $15.2,15.3$, and $15.4 \mathrm{MeV}$ were simulated. Local differences 

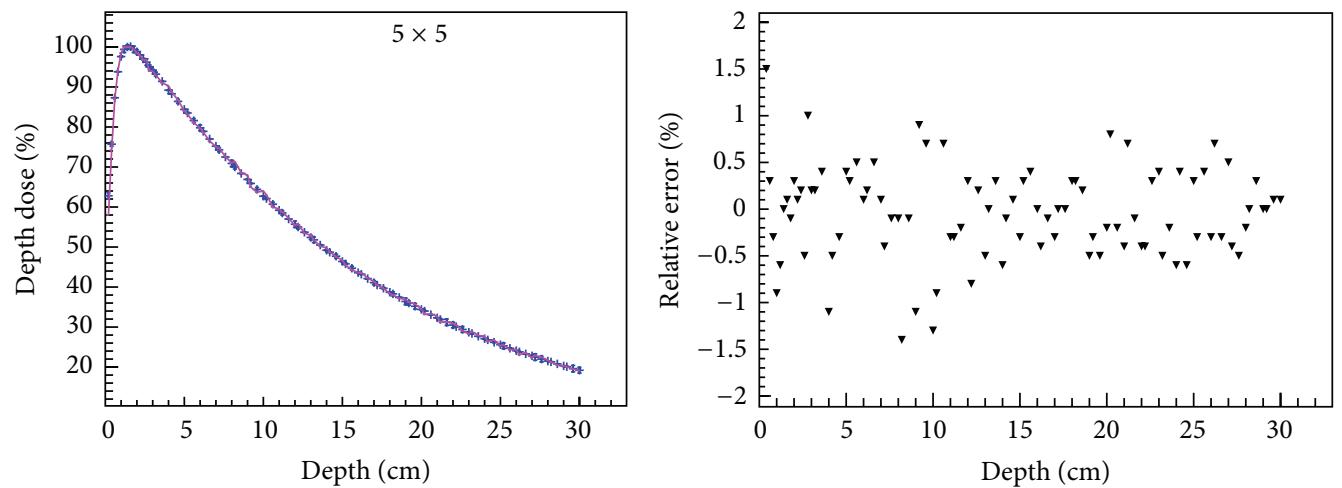

(a)
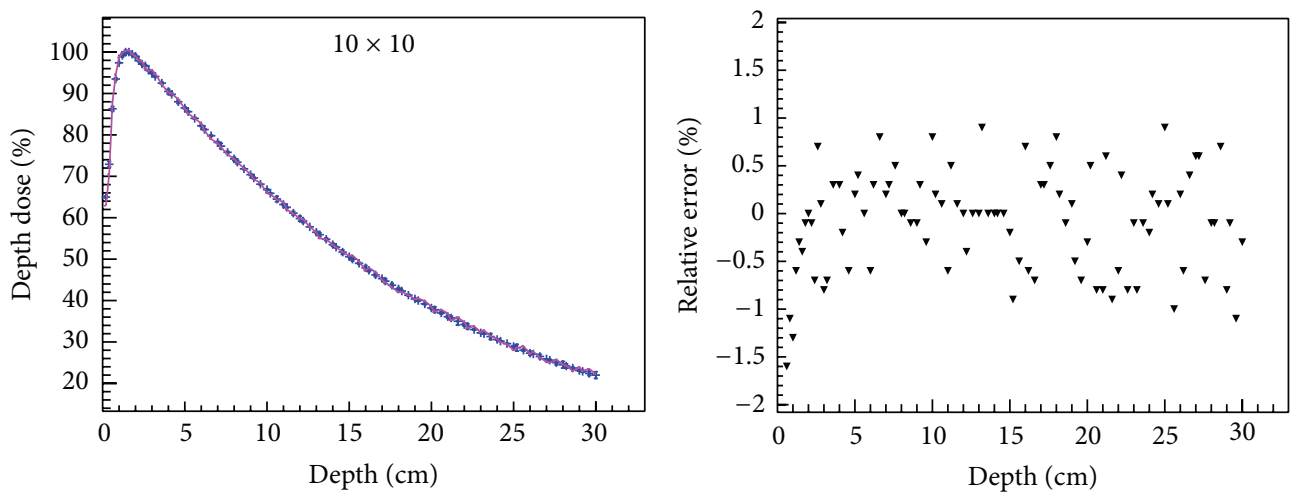

(b)
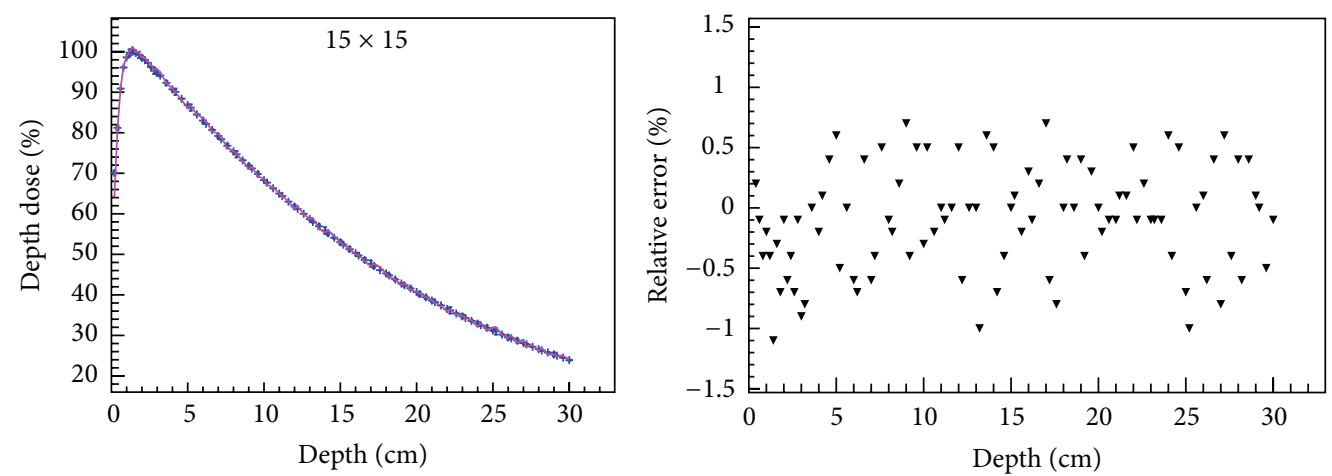

(c)
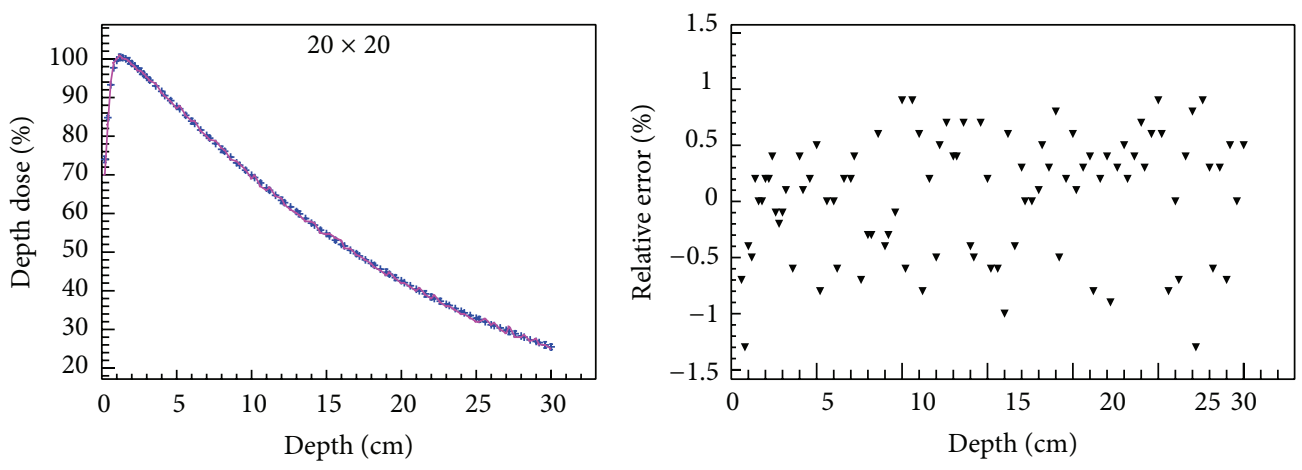

- Measurement

(d)

FIGURE 2: Comparison of calculated and measured percentage depth dose for $6 \mathrm{MV}$ photon beam for field sizes $5 \times 5 \mathrm{~cm}^{2}(\mathrm{a}), 10 \times 10 \mathrm{~cm}^{2}(\mathrm{~b})$, $15 \times 15 \mathrm{~cm}^{2}(\mathrm{c})$, and $20 \times 20 \mathrm{~cm}^{2}$ (d). Relative error is shown on right side. 

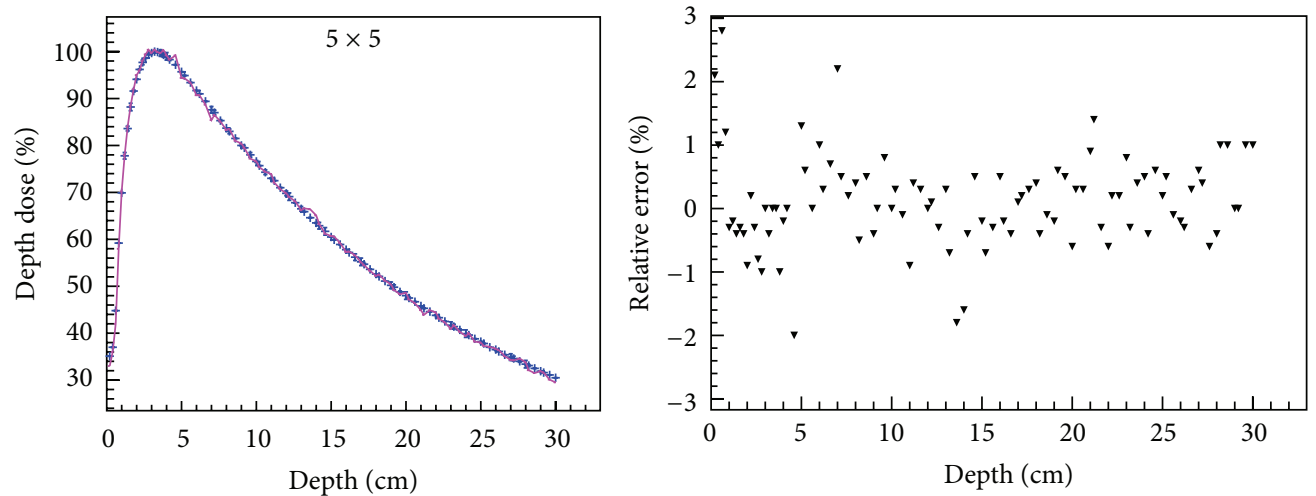

(a)
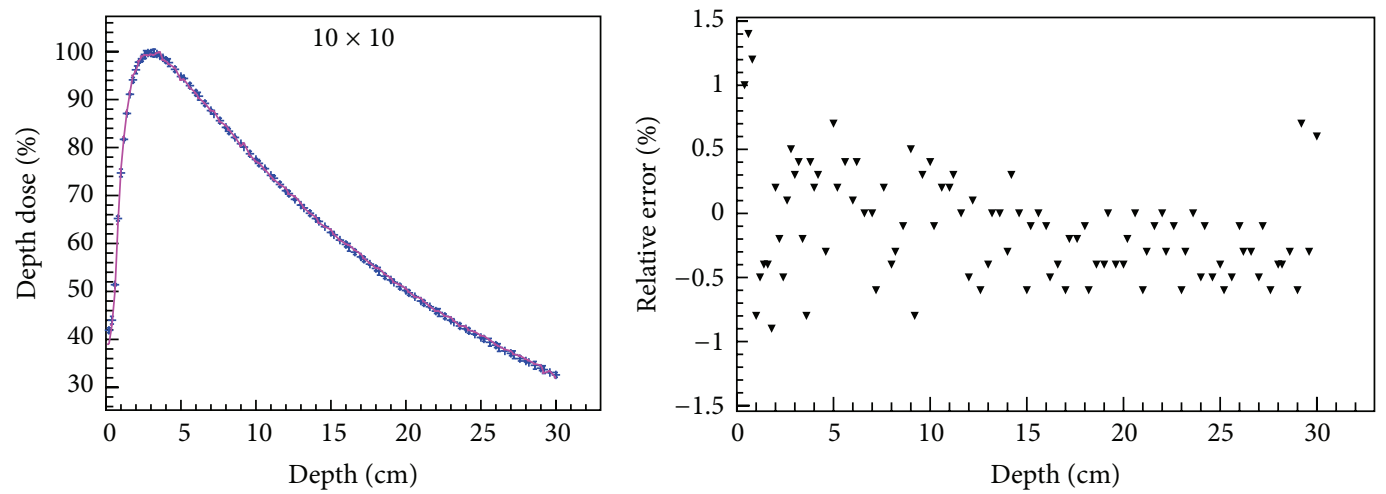

(b)
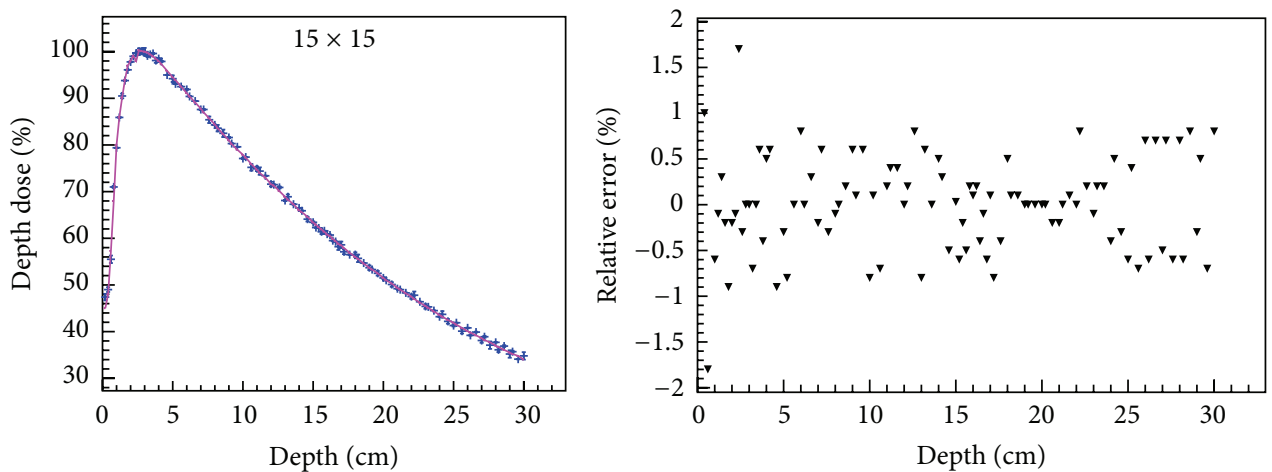

(c)
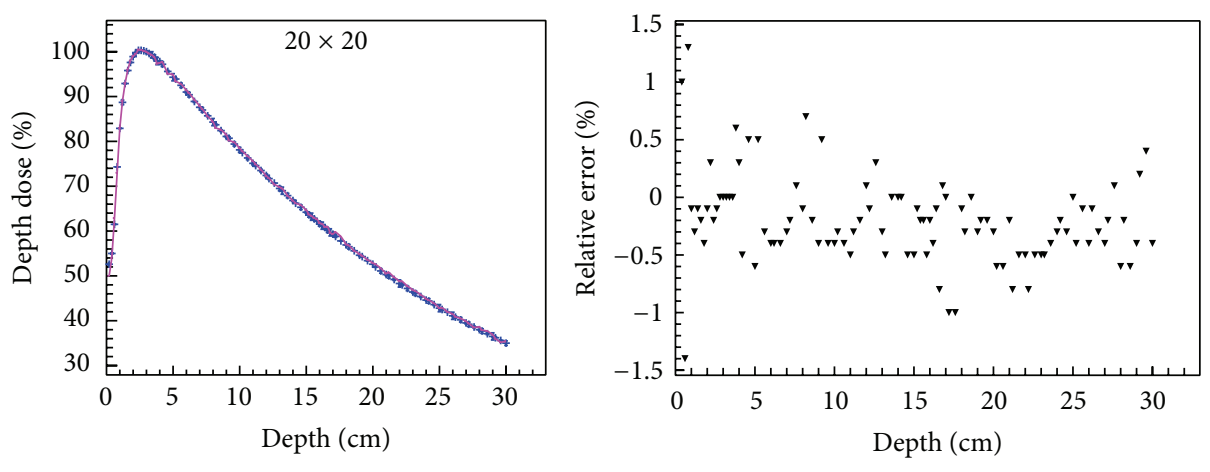

+ Measurement

— FLUKA

(d)

Figure 3: Comparison of calculated and measured percentage depth dose for $15 \mathrm{MV}$ photon beam for field sizes $5 \times 5 \mathrm{~cm}^{2}(\mathrm{a}), 10 \times 10 \mathrm{~cm}^{2}$ (b), $15 \times 15 \mathrm{~cm}^{2}$ (c), and $20 \times 20 \mathrm{~cm}^{2}$ (d). Relative error is shown on right side. 

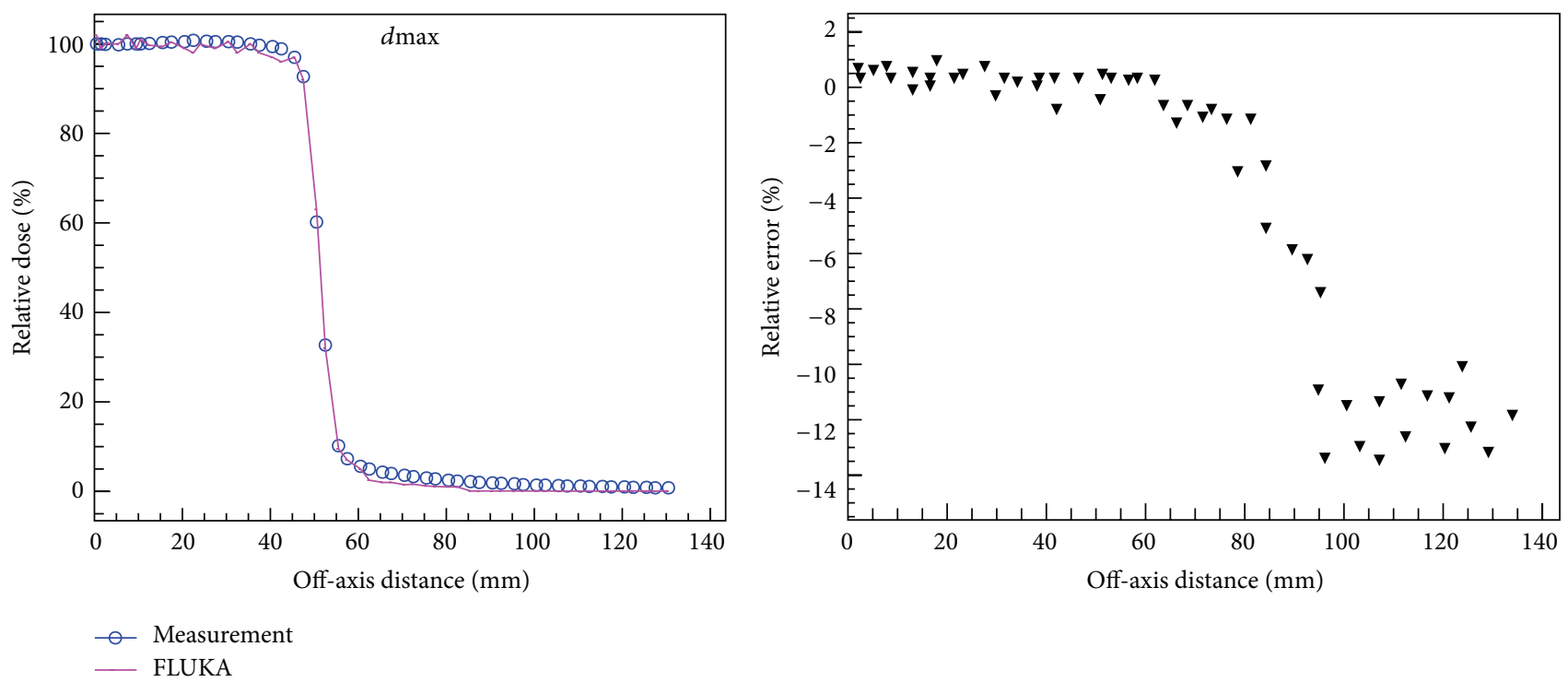

— FLUKA

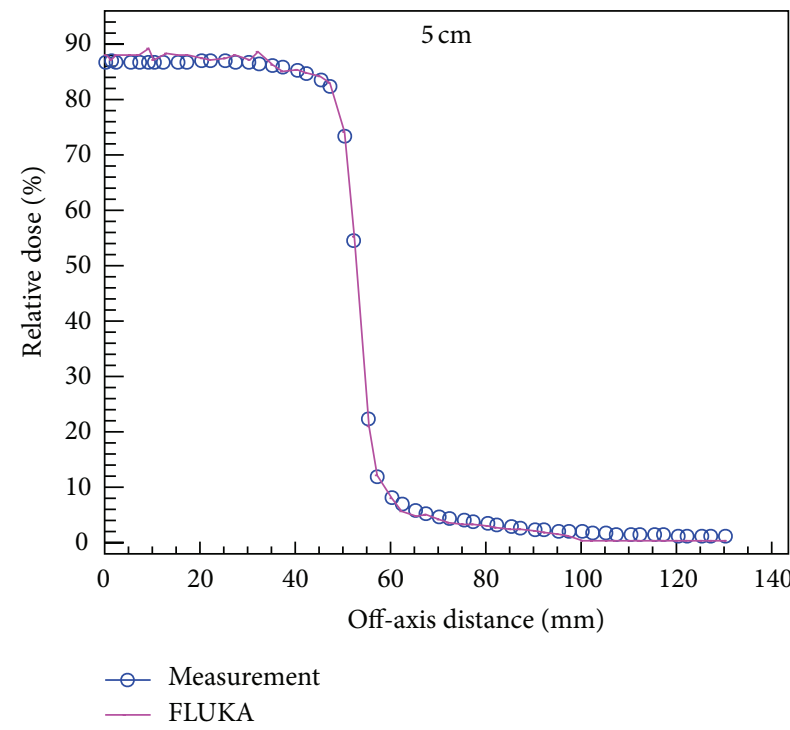

(a)

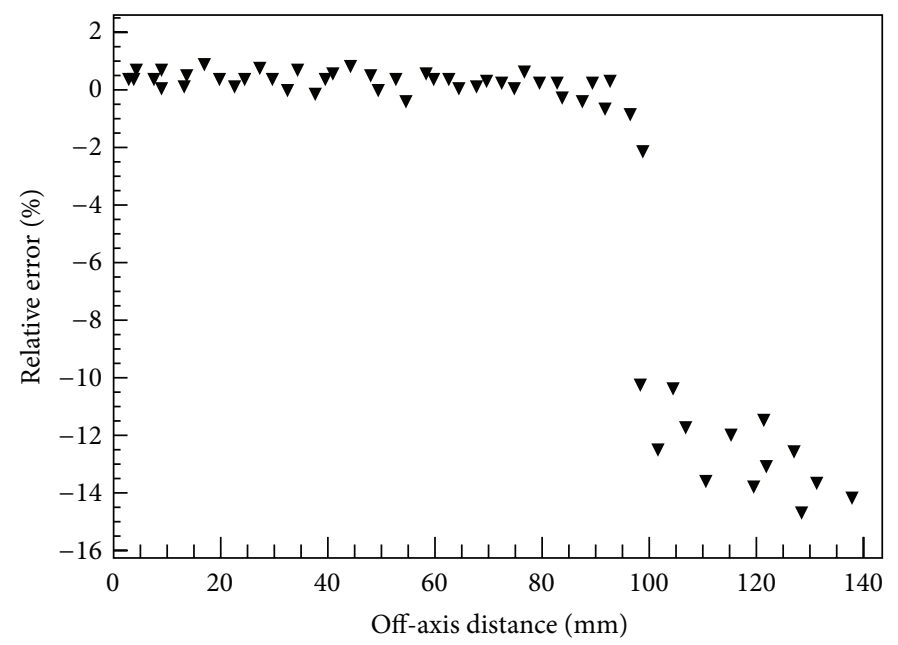

(b)

Figure 4: Comparison of calculated and measured depth dose profile and relative error (right side) for $6 \mathrm{MV}$ photon beam for depth of maximum dose (a) and $5 \mathrm{~cm}$ depths (b).

between simulation and measurement results were calculated for accurate comparison between results.

2.4. PDD and Beam Profile Comparison. Using the modeled dual energy linac, percentage depth dose and beam profiles were generated. PDD of both energies for $5 \times 5 \mathrm{~cm}^{2}, 10 \times$ $10 \mathrm{~cm}^{2}, 15 \times 15 \mathrm{~cm}^{2}$, and $20 \times 20 \mathrm{~cm}^{2}$ were generated using FLAIR of FLUKA Monte Carlo. This study estimates the accuracy and adequacy of our simplified model. The beam profiles of 6 and $15 \mathrm{MV}$ photon, including sharp dose fall off penumbra region (dose region between $80 \%$ and $20 \%$ ) for $10 \times 10 \mathrm{~cm}^{2}$ at depth of maximum dose and at $5 \mathrm{~cm}$ depth is compared in this study.

\section{Results and Discussion}

The Monte Carlo simulated percent depth dose curves and profiles across the central axis for flattened beams at different depths were compared against the measured data to verify the goodness of the MC model. The PDD curves are only sensitive to the mean energy of the incident electron, but dose profile curves are both dependent on the mean energy. Therefore, logically, one can use PDD to find the optimal energy. In our study $10 \times 10 \mathrm{~cm}^{2}$ field size is used to optimize the beam energy. The optimum beam energy for $6 \mathrm{MV}$ and $15 \mathrm{MV}$ clinical photon is found as $6.2 \mathrm{MeV}$ and $15.3 \mathrm{MeV}$. These values are largely dependent on the Monte Carlo models where linac head components are simulated. 

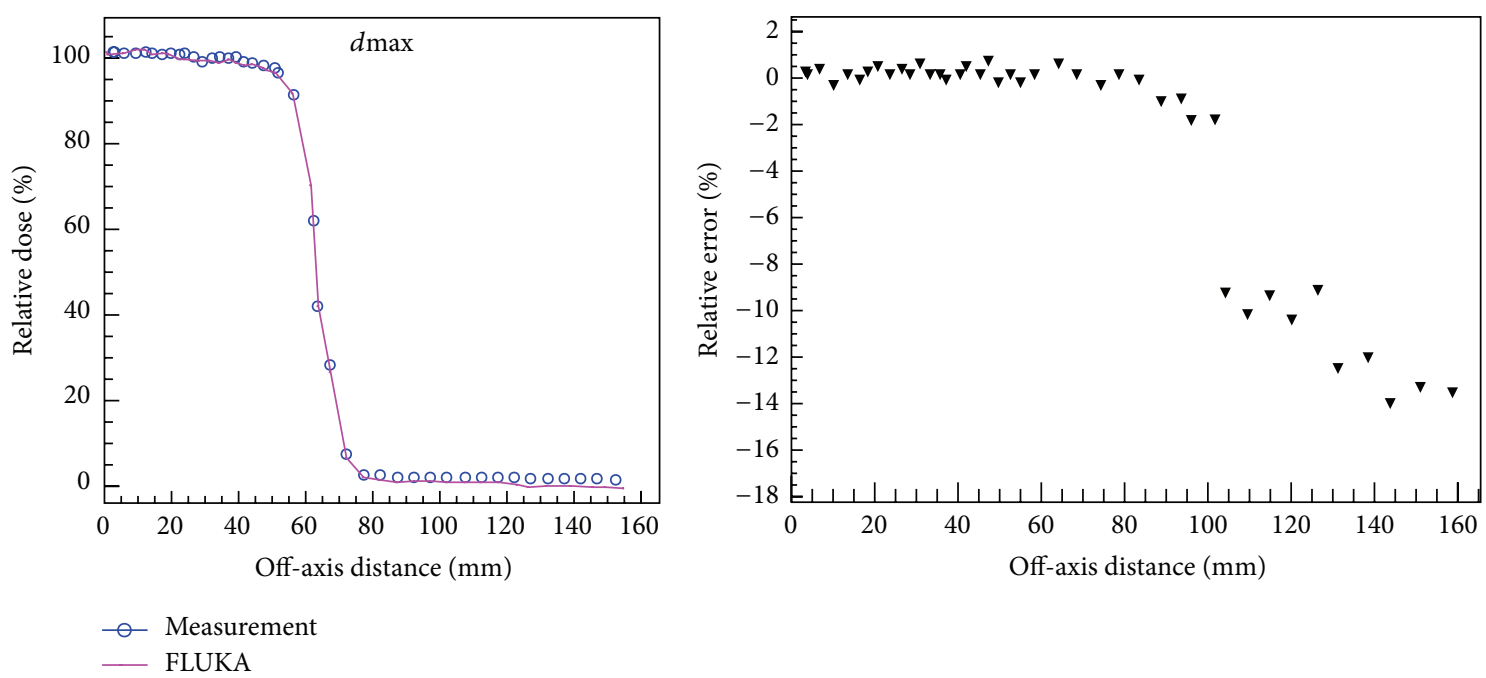

- FLUKA

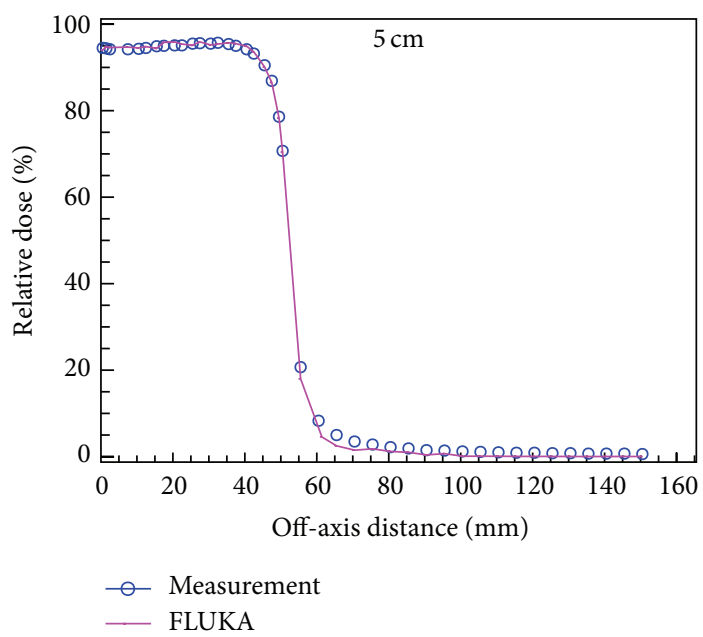

(a)

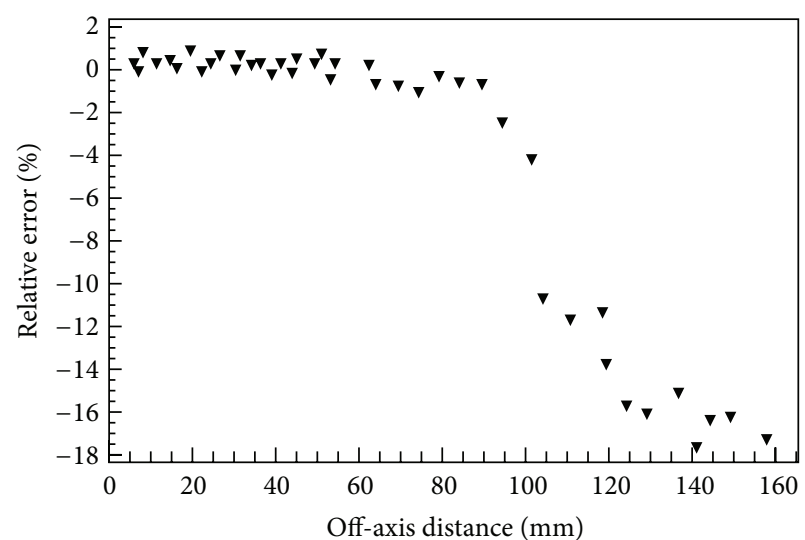

(b)

FIGURE 5: Comparison of calculated and measured depth dose profile and relative error (right side) for $15 \mathrm{MV}$ photon beam for depth of maximum dose (a) and $5 \mathrm{~cm}$ depths (b).

3.1. Depth Dose Curves Comparison. A comparison for the depth dose curves is shown in Figures 2 and 3. It can be concluded from the above figures that the Monte Carlo model for 6 and $15 \mathrm{MV}$ photon beam accurately matches the measured data, while central axis depth dose curves for the 6 and $15 \mathrm{MV}$ flattened beams for $5 \times 5 \mathrm{~cm}^{2}, 10 \times 10 \mathrm{~cm}^{2}, 15 \times$ $15 \mathrm{~cm}^{2}$, and $20 \times 20 \mathrm{~cm}^{2}$ show good agreement between measurement and simulation. Relative errors ((calculated measured)/calculated) over the depth are plotted separately in the previously mentioned figures. This small variation may be due to the changes in the geometry, especially in the truncated shape of flattening filter. Larger field sizes are not considered in this work due the limited use of such fields in our routine clinical practice.

3.2. Beam Profile Comparison. A cross-plane dose profiles comparison is shown in Figure 4 for $6 \mathrm{MV}$ and Figure 5 for $15 \mathrm{MV}$ photon beam. It is found that the Monte Carlo model has good agreement with the measured data from the previously mentioned figures. The flat region (within the field definition) is $2-3 \%$. The difference in the penumbra is about $10 \%$. The dose profile have a difference of $10-15 \%$ between data and simulation with in the field and high energy photon beam have a difference of $12 \%-18 \%$ in out-of-field (low dose) region. In the outside beam edge, our results were in agreement with previous studies $[15,16]$ where Monte Carlo predicts $20 \%-30 \%$ lower dose than the measurements.

\section{Conclusion}

This simplified FLUKA Monte Carlo model (avoiding the multileaf collimator) of Varian Clinac iX linac, based on the manufacturer's information, gives good agreement in percentage depth dose curves and beam profile plots. Monte Carlo model with retracted multileaf collimator shows perfect agreement for basic physical properties in standard field 
sizes. Although percentage depth dose values in the build-up region show more dependence on the field size (Figures 2 and 3 ), we commissioned calculation results for percentage depth dose curves and beam profiles (Figure 4) for different field sizes using recommended criteria for photon beam models. Measurements and simulation agrees better $(<2 \%)$ in the flat regions of beam profiles, while the out-of-field regions have a discrepancy of $10 \%-15 \%$.

\section{Conflict of Interests}

The authors declare no conflict of interests.

\section{Acknowledgments}

The authors sincerely acknowledge the assistance provided by Professor Roberto Cirio (INFN and University of Torino, Italy). The authors are grateful to Dr. Vincenzo Moncao (INFN and University of Torino, Italy) for his periodic suggestions and fruitful discussion.

\section{References}

[1] D. Sheikh-Bagheri and D. W. O. Rogers, "Sensitivity of megavoltage photon beam Monte Carlo simulations to electron beam and other parameters," Medical Physics, vol. 29, no. 3, pp. 379390, 2002.

[2] F. Verhaegen and J. Seuntjens, "Monte Carlo modelling of external radiotherapy photon beams," Physics in Medicine and Biology, vol. 48, no. 21, pp. R107-R164, 2003.

[3] A. Mesbahi, M. Fix, M. Allahverdi, E. Grein, and H. Garaati, "Monte Carlo calculation of Varian 2300C/D Linac photon beam characteristics: a comparison between MCNP4C, GEANT3 and measurements," Applied Radiation and Isotopes, vol. 62 , no. 3, pp. 469-477, 2005.

[4] A. Mesbahi, A. J. Reilly, and D. I. Thwaites, "Development and commissioning of a Monte Carlo photon beam model for Varian CL 2100EX linear accelerator," Applied Radiation and Isotopes, vol. 64, no. 6, pp. 656-662, 2006.

[5] T. D. Solberg, J. J. DeMarco, I. J. Chetty et al., "A review of radiation dosimetry applications using the MCNP Monte Carlo code," Radiochimica Acta, vol. 89, no. 4-5, pp. 337-355, 2001.

[6] M. K. Fix, P. Manser, E. J. Born, R. Mini, and P. Rüegsegger, "Monte Carlo simulation of a dynamic MLC based on a multiple source model," Physics in Medicine and Biology, vol. 46, no. 12, pp. 3241-3257, 2001.

[7] S. Y. Lin, T. C. Chu, and J. P. Lin, "Simulation of a clinical linear accelerator," Applied Radiation and Isotopes, vol. 55, pp. 759-765, 2001.

[8] S. B. Jiang, T. Pawlicki, and C. M. Ma, "Removing the effect of statistical uncertainty on dose-volume histograms from Monte Carlo dose calculations," Physics in Medicine and Biology, vol. 45 , no. 8, pp. 2151-2161, 2000.

[9] Y. Huang, R. A. Siochi, and J. E. Bayouth, "Dosimetric properties of a beam quality-matched $6 \mathrm{MV}$ unflattened photon beam," Journal of Applied Clinical Medical Physics, vol. 13, no. 4, pp. 7181, 2012.

[10] L. Wang, E. Yorke, G. Desobry, and C. Chui, "Dosimetric advantage of using $6 \mathrm{MV}$ over $15 \mathrm{MV}$ photons in conformal therapy of lung cancer: monte carlo studies in patient geometries," Journal of Applied Clinical Medical Physics, vol. 3, no. 1, pp. 51-59, 2002.

[11] G. Battistoni, S. Muraro, P. R. Sala et al., "The FLUKA code: description and benchmarking," in Proceedings of the Hadronic Shower Simulation Workshop, M. Albrow and R. Raja, Eds., vol. 896 of AIP Conference Proceeding, pp. 31-49, September 2007.

[12] A. Ferrari, P. R. Sala, A. Fasso, and J. Ranft, FLUKA: A Multiparticle Transport Code, CERN-2005-10, INFN/TC_05/11, SLACR-773, 2005.

[13] V. Vlachoudis, "FLAIR: a powerful but user friendly graphical interface for FLUKA," in Proceedings of the International Conference on Mathematics, Computational Methods Reactor Physics (M\&C ’09), Saratoga Springs, NY, USA, 2009.

[14] R. Brun and F. Rademakers, "ROOT-an object oriented data analysis framework," in Proceedings of the AIHENP Workshop on Nuclear Instruments and Methods in Physics Research A, pp. 81-86, Lausanne, Switzerland, September 1996, http://root.cern.ch/.

[15] J. Venselaar, H. Welleweerd, and B. Mijnheer, "Tolerances for the accuracy of photon beam dose calculations of treatment planning systems," Radiotherapy and Oncology, vol. 60, no. 2, pp. 191-201, 2001.

[16] C. L. H. Siantar, R. S. Walling, T. P. Daly et al., "Description and dosimetric verification of the PEREGRINE Monte Carlo dose calculation system for photon beams incident on a water phantom," Medical Physics, vol. 28, no. 7, pp. 1322-1337, 2001. 


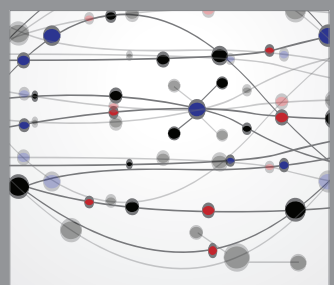

The Scientific World Journal
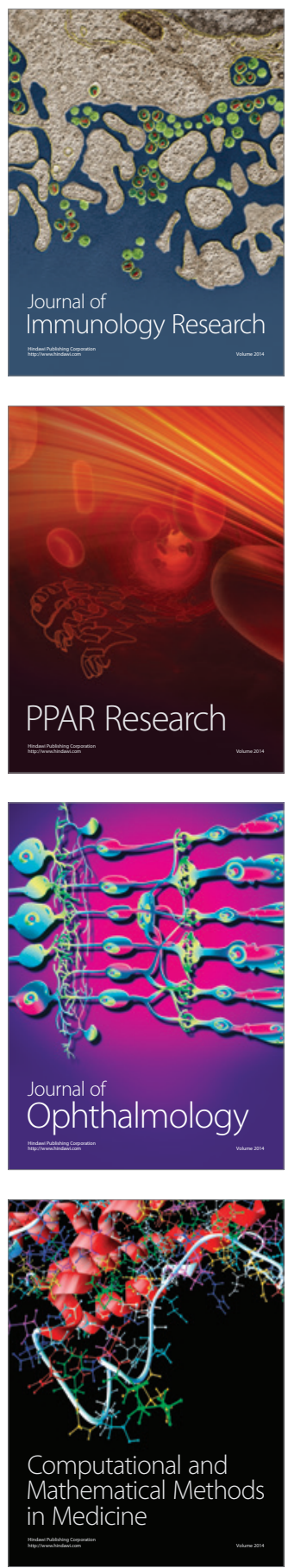

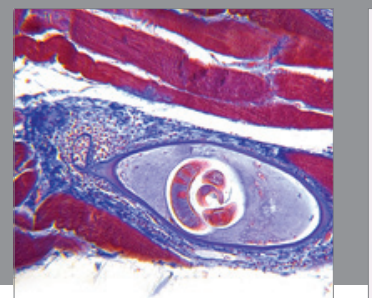

Gastroenterology

Research and Practice
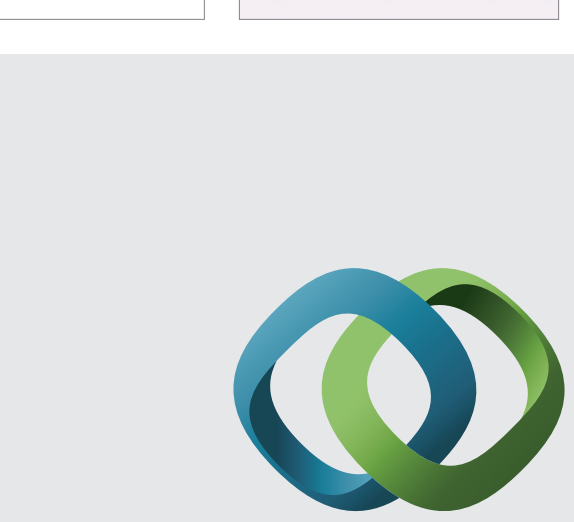

\section{Hindawi}

Submit your manuscripts at

http://www.hindawi.com
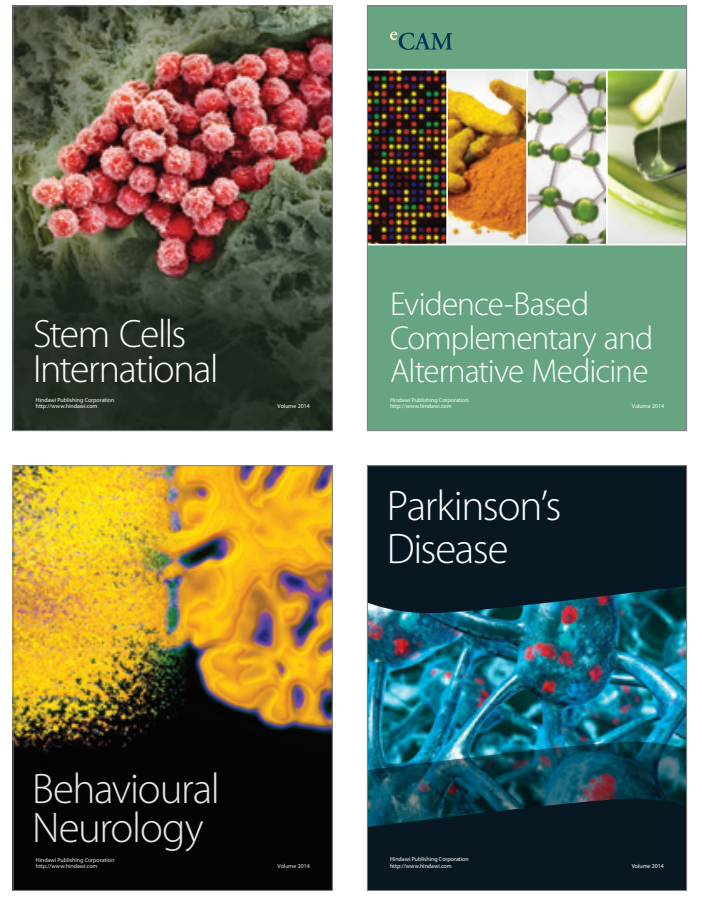
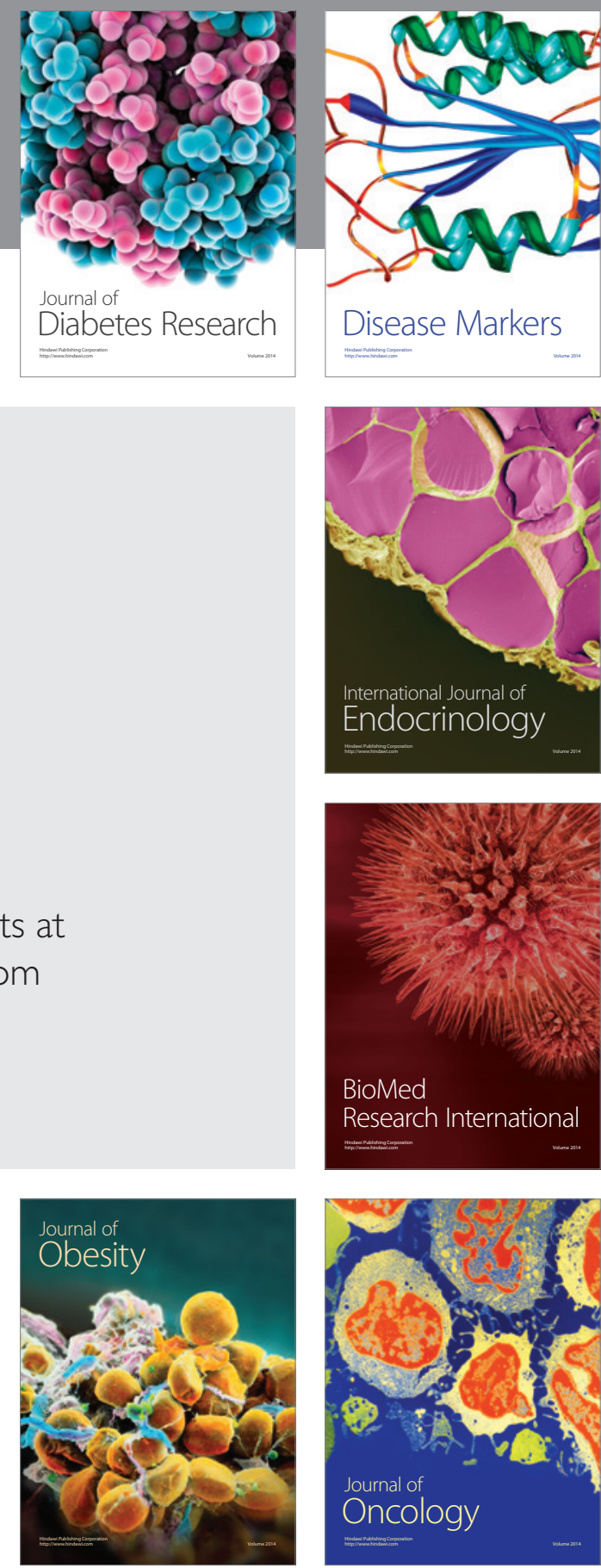

Disease Markers
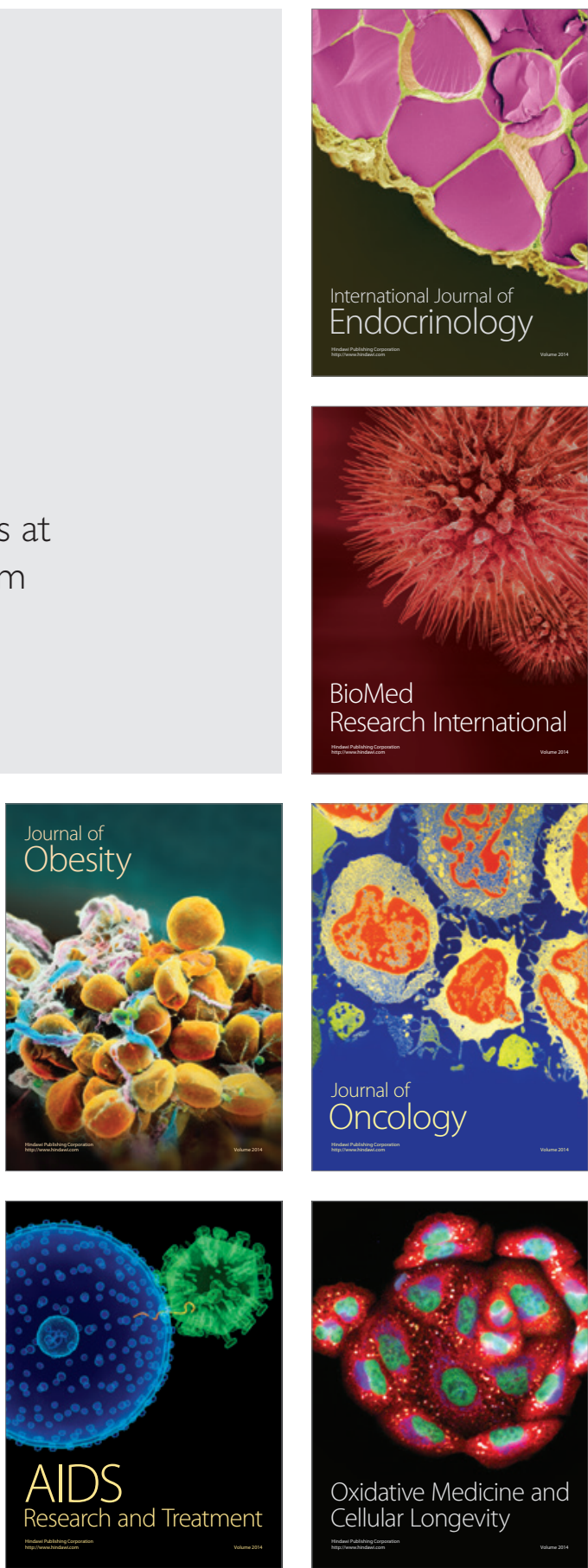